\title{
EXISTENCE OF POSITIVE SOLUTIONS TO A QUASILINEAR ELLIPTIC SINGULAR NEUMANN PROBLEM
}

\section{QING MiAO AND ZUODONG YANG}

Abstract. We show the existence of positive solution for the following singular Neumann problem

$$
\left\{\begin{array}{l}
-\operatorname{div}\left(|\nabla u|^{m-2} \nabla u\right)+\frac{a(x)}{u^{\beta}}=\lambda h(x) u^{p} \text { in } B_{R}, \\
\frac{\partial u}{\partial v}=0 \text { on } \partial B_{R},
\end{array}\right.
$$

where $R>0, \lambda>0$ is a positive parameter, $\beta>0, p \in[0, m-1)$. By means of double perturbation argument and variational methods, we obtain a positive solution $u \in C^{1}\left(\bar{B}_{R} \backslash\{0\}\right) \cap C\left(\bar{B}_{R}\right)$.

Mathematics subject classification (2010): 35J25, 35J65. conditions.

Keywords and phrases: quasilinear elliptic equation, sub-supersolution, variational method, Neumann

\section{REFERENCES}

[1] C. O. Alves, M. Montenegro, Positive solutions to a singular Neumann problem, J. Math. Anal. Appl., 352 (2009), 112-119.

[2] G. Astrita, G. Marrucci, Principles of non-Newtonian fluid mechanics, McGraw-Hill, 1974.

[3] H. Brezis, L. Nirenberg, Positive solutions of nonlinear elliptic equations involving critical Sobolev exponents, Comm. Pure Appl. Math., 36 (1983), 437-477.

[4] F. Cirstea, V. RAdulescu, Existence and uniqueness of positive solutions to a semilinear elliptic problem in $\mathbb{R}^{N}$, J. Math. Anal. Appl., 229 (1999), 417-425.

[5] M. G. Crandall, P. H. Rabinowitz, L. Tartar, On a Dirichlet problem with singular nonlinearity, Comm. Partial Differential Equations, 2 (1997), 193-222.

[6] J. I. Diaz, J. M. Morel, L. Oswald, An elliptic equation with singular nonlinearity, Comm. Partial Differential Equations, 12, 12 (1987), 1333-1344.

[7] A. EdELSON, Entire solutions of singular elliptic equations, J. Math. Anal. Appl., 139 (1989), $523-$ 532.

[8] Z. M. Guo, Some existence and multiplicity results for a class of quasilinear elliptic eigenvalue problems, Nonlinear Anal., 18 (1992), 957-971.

[9] Z. M. Guo, Existence and uniqueness of positive radial solutions for a class of quasilinear elliptic equations, Appl. Anal., 47 (1992), 173-190.

[10] Z. M. Guo, Boundary value problems of a class of quasilinear ordinary differential equations, Differential Integral Equations, 6, 3 (1993), 705-719.

[11] Z. M. GUO, On the number of positive solutions for quasilinear elliptic eigenvalue problems, Nonlinear Anal., 27, 2 (1996), 229-247.

[12] Z. M. GUO, J. R. L. WeBB, Uniqueness of positive solutions for quasilinear elliptic equations when a parameter is large, Proc. Roy. Soc. Edinburgh. Sect. A, 124 (1994), 189-198.

[13] Z. M. GUO, J. R. L. WEBB, Large and small solutions of a class of quasilinear elliptic eigenvalue problems, J. Differential Equations, 180 (2002), 1-50.

[14] A. S. KALASHNIKOV, On a nonlinaer equation appearing in the theory of non-stationary filtration, Trudy Sem. Petrovsk., 4 (1978), 137-146.

[15] T. KusAno, C. A. SWANSOn, Entire positive solutions of singular semilinear elliptic equations, Japan J. Math., 11 (1985), 145-155. 
[16] A. C. LAZER, P. J. MCKenna, On a singular nonlinear elliptic boundary value problem, Proc. Amer. Math. Soc., 111, 3 (1991), 721-730.

[17] A. V. LAIR, A. W. SHAKER, Entire solution of a singular semilinear elliptic problem, J. Math. Anal. Appl., 200 (1996), 498-505.

[18] Q. S. LU, Z. D. YANG, E. H. TwIZELL, Existence of entire explosive solutions of quasilinear elliptic equations, Appl. Math. Comput., 148 (2004), 359-372.

[19] L. K. MARTINSON, K. B. PAVLOv, Unsteady shear flows of a conducting fluid with a rheological power law, Magnitnaya Gidrodinamika, 2 (1971), 50-58.

[20] Q. Miao, Z. D. Yang, Bounded Positive Entire Solutions of Singular p-Laplacian Equations, Nonlinear Anal., 69 (2008), 3749-3760.

[21] M. Del Pino, G. Hernandez, Solvability of the Neumann problem in a ball for $-\Delta u+u^{-v}=$ $h(|x|), v>1$, J. Differential Equations, 124 (1996), 108-131.

[22] O. S. DE QUEIROZ, A Neumann problem with logarithmic nonlinearity in a ball, Nonlinear Anal., 70 (2009), 1656-1662.

[23] B. J. XuAn, Z. C. Chen, Solvability of singular quasilinear elliptic equation, Chinese Ann. Math. Ser. A, 20, 1 (1999), 117-128.

[24] Z. D. YANG, Non-existence of positive entire solutions for elliptic inequalities of p-laplacian, Appl. Math. J. Chinese Univ. Ser. B, 12, 4 (1997), 399-410.

[25] Z. D. YANG, Existence of entire explosive positive radial solutions for a class of quasilinear elliptic systems, J. Math. Anal. Appl., 288, 2 (2003), 768-783.

[26] Z. D. YANG, Existence of positive entire solutions for singular and non-singular quasi-linear elliptic equation, J. Comput. Appl. Math., 197, 2 (2006), 355-364.

[27] Z. D. YANG, Q. S. LU, Blow-up estimates for a quasilinear reaction-diffusion system, Math. Methods Appl. Sci., 26 (2003), 1005-1023.

[28] Z. D. YANG, Q. S. LU, Nonexistence of positive solutions to a quasilinear elliptic system and blow-up estimates for a quasilinear reaction-diffusion system, J. Comput. Appl. Math., 50 (2003), 37-56. 